\title{
Mechanisms of microRNA-142 in mitochondrial autophagy and hippocampal damage in a rat model of epilepsy
}

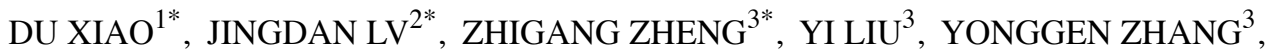 \\ CUIZHU LUO ${ }^{3}, \mathrm{LIU}^{4} \mathrm{QI}^{4}, \mathrm{BING} \mathrm{QIN}^{4}$ and $\mathrm{CHAO} \mathrm{LIU}^{5}$ \\ ${ }^{1}$ Department of Obstetrics and Gynecology, Guangzhou Women and Children's Medical Center, \\ Guangzhou Medical University, Guangzhou, Guangdong 510623; ${ }^{2}$ Department of Neurology, Guangzhou Hospital of TCM, \\ Guangzhou Medical University, Guangzhou, Guangdong 510130; ${ }^{3}$ Department of Intensive Care Unit, \\ Pingxiang People's Hospital of Southern Medical University, Pingxiang, Jiangxi 337055; \\ ${ }^{4}$ Epilepsy Center and Department of Neurosurgery, The First Affiliated Hospital, Jinan University, \\ Guangzhou, Guangdong 510623; ${ }^{5}$ Department of Neurology, The First Hospital of Changsha, \\ Changsha, Hunan 410013, P.R. China
}

Received February 3, 2020; Accepted December 16, 2020

DOI: $10.3892 /$ ijmm.2021.4931

\begin{abstract}
Researchers have confirmed the microRNA (miRNA/miR)-epilepsy association in rodent models of human epilepsy via a comprehensive database. However, the mechanisms of miR-142 in epilepsy have not been extensively studied. In the present study, a rat model of epilepsy was first established by an injection of lithium chloride-pilocarpine and the successful establishment of the model was verified via electroencephalogram monitoring. The levels of miR-142, phosphatase and tensin homolog deleted on chromosome 10 (PTEN)-induced putative kinase 1 (PINK1), marker proteins of mitochondrial autophagy, and apoptosis-related proteins were measured. Additionally, the pathological changes in the hippocampus, the ultrastructure of the mitochondria, and degeneration and the apoptosis of neurons were observed using different staining methods. The malondialdehyde (MDA) content and superoxide dismutase (SOD) activity in the hippocampus, mitochondrial membrane potential (MTP) and reactive oxygen species (ROS) generation were detected. Furthermore, the targeting association between miR-142 and
\end{abstract}

Correspondence to: Dr Chao Liu, Department of Neurology, The First Hospital of Changsha, 311 Yinpan Road, Changsha, Hunan 410013, P.R. China

E-mail: liuchao_0710@163.com

Dr Du Xiao, Department of Obstetrics and Gynecology, Guangzhou Women and Children's Medical Center, Guangzhou Medical University, 402 Renmin Zhong Road, Liwan, Guangzhou, Guangdong 510623, P.R. China

E-mail:wvan7471khlx@163.com

${ }^{*}$ Contributed equally

Key words: epilepsy, microRNA-142, PTEN-induced putative kinase 1 , mitochondrial autophagy, hippocampal damage
PINK1 was predicted and verified. Consequently, apoptosis increased, and mitochondrial autophagy decreased, in the hippocampus of epileptic rats. Following miR-142 inhibition, the epileptic rats exhibited an increased Bax expression, a decreased $\mathrm{Bcl}-2$ expression, upregulated marker protein levels of mitochondrial autophagy, a reduced MDA content, an enhanced SOD activity, an increased MTP and decreased ROS generation. PINK1 is a target gene of miR-142, and its overexpression protected against hippocampal damage. Taken together, the results of the present study demonstrated that miR-142 inhibition promotes mitochondrial autophagy and reduces hippocampal damage in epileptic rats by targeting PINK1. These findings may provide useful information for the treatment of epilepsy.

\section{Introduction}

Epilepsy is a prevalent brain disease characterized by long-term vulnerability to seizures and emotional and cognitive impairment, affecting approximately 50 million individuals worldwide (1). Approximately $40 \%$ of epileptic patients have structural or metabolic causes due to different brain injuries (2) and develop depression and anxiety (3). Previous research has indicated that the incidence of epilepsy in developing nations is significantly higher than that in developed countries (4). Recently, the average annual cost of each epilepsy patient was reported to be approximately 5,253 USD in China, accounting for more than half of their annual income (5). Additionally, it has been scientifically confirmed that factors, such as chronic stress, brain abnormalities and interactions between underlying genetic factors and the environment may lead to epilepsy $(6,7)$. Head injury, infection, trauma, malnutrition, and poor prenatal and perinatal care are associated with an increased risk of seizures and epilepsy (4). Additionally, the abnormal accumulation of damaged mitochondria in the central nervous system, particularly in the hippocampus, has been reported to contribute to mitochondrial autophagy defects and may be 
involved in the pathology of epilepsy (8). Therefore, the present study focused on mitochondrial autophagy to investigate the underlying molecular mechanisms in epilepsy.

MicroRNAs (miRNAs or miRs) are a class of small, well-preserved noncoding RNAs that monitor numerous biological processes and cellular functions during the development of the central nervous system, and the dysfunction of miRNAs contributes to neurological diseases $(9,10)$. miR-142 is a highly conservative miR in many invertebrates and vertebrates, and it has been widely studied in the hematopoietic system, cancers, the inflammatory response and immune tolerance (11). Junker et al reported that miR-142 expression was more abundant in active multiple sclerosis brain lesions than in normal white matter, suggesting that miR-142 may be involved in brain inflammatory and degenerative diseases (12). However, the role of miR-142 in epilepsy has not been extensively studied. In the present study, it was found that miR-142 could target phosphatase and tensin homolog deleted on chromosome 10 (PTEN)-induced putative kinase 1 (PINK1). PINK1 is a mitochondrial targeting kinase that accumulates on damaged mitochondria and protects cells from stress-induced mitochondrial dysfunction (13). As a neuroprotective kinase, PINK1 is processed by healthy mitochondria and is released to trigger neuron differentiation (14). PTEN deficiency can lead to severe brain dysfunction, including ataxia, seizures and brain enlargement (15). However, the mechanisms of PINK1 in epilepsy remain largely unknown. Considering the above-mentioned findings, the present study examined the effects of miR-142 and PINK1 on mitochondrial autophagy in a rat model of epilepsy.

\section{Materials and methods}

Animal treatment and grouping. The study was approved and supervised by the Ethics Committee of Guangzhou Women and Children's Medical Center. The protocol was also approved by the Institutional Animal Care and Use Committee of Guangzhou Women and Children's Medical Center. Significant efforts were made to minimize the number of animals used and their pain. A total of 96 healthy male Wistar rats (6-8 weeks old, weighing $220 \pm 20 \mathrm{~g}$ ) purchased from Dongzhimen Hospital, Beijing University of Chinese Medicine [Certificate no. SYXK (Jing) 2015-0001, Beijing, China] were raised under specific pathogen-free conditions at a constant temperature $\left(20 \pm 2^{\circ} \mathrm{C}\right)$ and humidity (50-60\%) with free access to sterilized drinking water and standard feed. The rats were randomly split into the normal group, epilepsy group, antagomir-negative control (NC) group, antagomir-miR-142 group, lentivirus vector (LV)-NC group, LV-PINK1 group, antagomir-miR-142 + siRNA-NC group, and antagomir-miR-142 + siRNA-PINK1 group, with 12 rats in each group. Following the establishment of the model of epilepsy, the rats were administered a corresponding stereotaxic injection of antagomir-NC, antagomir-miR-142, LV-NC, LV-PINK1, antagomir-miR-142 + siRNA-NC and antagomir-miR-142 + siRNA-PINK1 into the brain as described below. The injectants were all from Shanghai GenePharma Co., Ltd.

Establishment of model of epilepsy. The rats were administered $125 \mathrm{mg} / \mathrm{kg}$ of lithium chloride via intraperitoneal injection, followed by $20 \mathrm{mg} / \mathrm{kg}$ of pilocarpine (both from
Sigma-Aldrich; Merck KGaA) 18-20 h later, as previously described (16). At $30 \mathrm{~min}$ prior to the pilocarpine administration, the rats were administered $1 \mathrm{mg} / \mathrm{kg}$ of atropine (Sigma-Aldrich; Merck KGaA) to alleviate the peripheral cholinergic effect of pilocarpine. Rats in the normal group were injected with an equal amount of saline. Following observation for 30 min, according to the Racine (17) grading standard, the model of epilepsy was successfully established when the seizure activity reached grade IV or above and lasted for $>30 \mathrm{~min}$. To control the seizure intensity and reduce mortality, $10 \mathrm{mg} / \mathrm{kg}$ of diazepam (Sigma-Aldrich; Merck KGaA) was injected intraperitoneally $90 \mathrm{~min}$ following the onset of the epilepticus status. The electroencephalogram (EEG, Medelec) data of the rats were recorded before epilepsy and at $3 \mathrm{~h}$ after epilepsy; the data of normal rats were also collected.

Stereotaxic injection into the rat brain. Epileptic rats were anesthetized with $1 \%$ pentobarbital sodium $(45 \mathrm{mg} / \mathrm{kg})$ through intraperitoneal injection. The incisor teeth of the rats were fixed using a maxillary fixator of a stereotaxic instrument (Shanghai Medical Instruments Co., Ltd.). Subsequently, 1 ear stick was pushed into the external auditory canal of the rats to locate the rat's head in the middle of the 2 slip paths, and the ear stick on the other side was pushed in to fix the head. The scalp of the rats was shaved to determine the bregma of the stereotaxic localization of the brain. The right lateral ventricle was determined as the injection site (AP, $-1 \mathrm{~mm}$; LL, $1.5 \mathrm{~mm}$; V, $-4.5 \mathrm{~mm}$ ), and $10 \mu \mathrm{l}$ antagomir-NC (4 nmol), antagomir-miR-142 (4 nmol) (18), LV-NC $\left(6 \times 10^{4} \mathrm{TU}\right)$, LV-PINK1 $\left(6 \times 10^{4} \mathrm{TU}\right)$, antagomir-miR-142 + siRNA-NC $(0.5 \mu \mathrm{g})$ or antagomir-miR-142 + siRNA-PINK1 $(0.5 \mu \mathrm{g})$ were injected into the brains of the rats at $0.25 \mu 1 / \mathrm{min}$ using a Hamilton syringe (Hamilton Company). After the injection, the needle was pulled out; the skull was sealed with bone wax and the skin was sutured and disinfected. The rats were allowed to recover for 5 days after the injection. During this period, the health status of the rats was detected every day, and no deaths occurred. Following recovery, samples were collected for use in subsequent experiments.

Tissue collection. Following anesthesia $(45 \mathrm{mg} / \mathrm{kg}, 1 \%$ pentobarbital sodium through intraperitoneal injection), the rats were rapidly decapitated, and the hippocampal tissues were rapidly separated from the ice. In each group, 3 hippocampal tissues were made into a homogenate and stored at $-80^{\circ} \mathrm{C}$ until analysis, and another 3 hippocampal tissues $\left(1 \mathrm{~mm}^{3}\right)$ were used for transmission electron microscope (TEM) observation, and the remaining 6 hippocampal tissues were fixed with $10 \%$ formalin for $24 \mathrm{~h}$. The hippocampal tissues were dehydrated, sliced into $4-\mu \mathrm{M}$-thick sections, and were then embedded in paraffin for tissue staining.

Reverse transcription-quantitative polymerase chain reaction (RT-qPCR). Total RNA was extracted from the tissue samples using the TRIzol kit (Invitrogen; Thermo Fisher Scientific, Inc.) and was then reverse transcribed into cDNA using the Rever Tra Ace ${ }^{\circledR}$ aPCR RT Master Mix (Toyobo Co., Ltd.). The designed primers were synthesized by Takara Holdings Inc. (Table I). Reverse transcription was performed in a $10-\mu \mathrm{l}$ volume in a $37^{\circ} \mathrm{C}$ water bath for $15 \mathrm{~min}$, followed 
Table I. Sequences of primers used for RT-qPCR.

\begin{tabular}{ll}
\hline Gene & \multicolumn{1}{c}{ Sequence (5'-3') } \\
\hline miR-142 & F: 5'-GGCGCTAGCAAGAGAGGGG-3' \\
& R: 5'-GGCGGATCCTCGTCCTTGACGCTG-3' \\
U6 & F: 5'-CTCGCTTCGGCAGCACA-3' \\
& R: 5'-AACGCTTCACGAATTTGCGT-3' \\
PINK1 & F: 5'-ATCAGTAGCATCTAGCATAC-3' \\
& R: 5'-GATCACTGATCAGATCTATCC-3' \\
GAPDH & F: 5'-TCTCCCTCACAATTTCCATCCC-3' \\
& R: 5'-TTTTTGTGGGTGCAGCGAAC-3'
\end{tabular}

RT-qPCR, reverse transcription quantitative polymerase chain reaction; F, forward; R, reverse; miR-142, microRNA-142; PINK1, phosphatase and tensin homolog deleted on chromosome 10 (PTEN)-induced putative kinase 1; GAPDH, glyceraldehyde-3-phosphate dehydrogenase.

by incubation at $50^{\circ} \mathrm{C}$ for $5 \mathrm{~min}$ and then incubation at $98^{\circ} \mathrm{C}$ for $5 \mathrm{~min}$ for reverse transcriptase inactivation according to the manufacturer's instructions. The reaction solution was subjected to quantitative fluorescence following the instructions of the $\mathrm{SYBR}^{\circledR}$ Premix Ex $\mathrm{Taq}^{\mathrm{TM}}$ II kit in the ABI PRISM $^{\circledR} 7300$ system. The $50-\mu 1$ reaction system comprised $25 \mu$ l of SYBR ${ }^{\circledR}$ Premix Ex Taq ${ }^{\mathrm{TM}}$ II $(2 \mathrm{X}), 2 \mu \mathrm{l}$ of PCR forward primers, $2 \mu \mathrm{l}$ of PCR reverse primers, $1 \mu 1$ of ROX Reference Dye $(50 \mathrm{X}), 4 \mu \mathrm{l}$ of DNA template and $16 \mu \mathrm{l}$ of $\mathrm{ddH}_{2} \mathrm{O}$. The reaction conditions were as follows: Pre-denaturation at $95^{\circ} \mathrm{C}$ for $5 \mathrm{~min}$, and 40 cycles of denaturation at $95^{\circ} \mathrm{C}$ for $40 \mathrm{sec}$, annealing at $60^{\circ} \mathrm{C}$ for $30 \mathrm{sec}$, and extension at $72^{\circ} \mathrm{C}$ for $1 \mathrm{~min}$. U6 served as the internal reference for miR-142 and glyceraldehyde-3-phosphate dehydrogenase (GAPDH) for PINK1. The relative transcription levels of target genes were calculated using the $2^{-\Delta \Delta \mathrm{Ct}}$ relative quantitative method (19). $\Delta \Delta \mathrm{Ct}=\Delta \mathrm{Ct}$ epilepsy group- $\Delta \mathrm{Ct}$ control group, $\Delta \mathrm{Ct}=\mathrm{Ct}$ (target gene) $-\mathrm{Ct}$ (internal reference).

Western blot analysis. Total proteins were extracted from the hippocampal tissues according to the instructions provided with TRIzol reagent (Invitrogen; Thermo Fisher Scientific, Inc.). The protein concentration was measured and adjusted with deionized water to ensure an equal loading amountu sing the bicinchoninic acid assay kit (Jiancheng Bioengineering Institute). Subsequently, 10\% separating gels and stacking gels were prepared. The samples were mixed with the loading buffer, boiled at $100^{\circ} \mathrm{C}$ for $5 \mathrm{~min}$, cooled on ice, centrifuged at $15,000 \times \mathrm{g}$ and $4^{\circ} \mathrm{C}$ for $5 \mathrm{~min}$, and loaded into each lane at equal amounts using a pipette for electrophoretic separation. Subsequently, the proteins $(40 \mu \mathrm{g})$ on the gel were transferred to nitrocellulose membranes and blocked with $5 \%$ non-fat dry milk at $4^{\circ} \mathrm{C}$ overnight. Thereafter, the membranes were incubated with the primary antibodies (all from Abcam Inc.) PINK1 (ab23707; 1:1,000), translocase of the inner membrane 23 (TIM23; ab230253; 1:1,000), C-III core $1(\mathrm{ab} 2740 ; 1 \mu \mathrm{g} / \mathrm{ml})$, cytochrome $c$ oxidase IV (COX IV; ab33985; $1 \mu \mathrm{g} / \mathrm{ml}$ ), mitochondrial fusion protein 1 (MFN1; ab104274; $1 \mu \mathrm{g} / \mathrm{ml}$ ), B-cell lymphoma-2 (Bcl-2; ab182858; 1:2,000) and Bcl-2-associated X (Bax; ab32503;
$1: 1,000)$ at $4^{\circ} \mathrm{C}$ overnight. The membranes were then washed with phosphate-buffered saline (PBS) at room temperature 3 times, each for $5 \mathrm{~min}$. Subsequently, the membranes were incubated with the horseradish peroxidase-labeled secondary antibody immunoglobulin G (1:1,000; ab6702, or 1:3,000; ab6708; Abcam) at $37^{\circ} \mathrm{C}$ for $1 \mathrm{~h}$ and then were washed with PBS again at room temperature 3 times, each for $5 \mathrm{~min}$. The membranes were then rinsed in enhanced chemiluminescence reagent (Pierce; Thermo Fisher Scientific, Inc.) at room temperature for $1 \mathrm{~min}$. Following liquid removal, the membranes were covered with food cling wraps, exposed in the dark, developed and fixed to observe the results. GAPDH was used as an internal reference. The protein marker was purchased from Pierce; Thermo Fisher Scientific, Inc. The western blots were analyzed using ImageJ2x V2.1.4.7 (Rawak Software, Inc.).

Hematoxylin and eosin $(H \& E)$ staining. Tissue sections were dewaxed in xylene I and II for $5 \mathrm{~min}$ and were then separately rinsed in 100, 95, 80 and $75 \%$ ethanol for $2 \mathrm{~min}$, and then in PBS $(2 \times 5 \mathrm{~min})$. The sections were then stained with hematoxylin at room temperature for $5 \mathrm{~min}$, washed in running water for $3 \mathrm{~min}$, color-separated with $1 \%$ hydrochloric ethanol for $10 \mathrm{sec}$, and turned to blue with $1 \%$ ammonia for $30 \mathrm{sec}$. Following a 2-min wash in running water, the sections were rinsed in 50, 70 and $80 \%$ ethanol for $2 \mathrm{~min}$ and were then stained with eosin at room temperature for $2 \mathrm{~min}$. Thereafter, the sections were washed in running water for $3 \mathrm{~min}$, rinsed in $95 \%$ ethanol, absolute ethanol I and II for $3 \mathrm{~min}$ each, followed by rinsing in xylene I and II for $5 \mathrm{~min}$ each. Finally, the sections were sealed with neutral gum and observed under a BX-51 optical microscope (Olympus Optical Co., Ltd.) by two pathologists in a blinded manner.

Nissl staining. Paraffin-embedded sections were dewaxed with $0.5 \%$ cresyl violet at room temperature, hydrated and stained with Nissl dye (G1434; Beijing Solarbio Science \& Technology Co., Ltd.) at room temperature for $10 \mathrm{~min}$. The sections were dehydrated and sealed. Finally, each section was observed by 2 pathologists under a BX-51 optical microscope (Olympus Optical Co., Ltd.) and the Nissl-positive cells were counted.

Terminal deoxynucleotidyl transferase (TdT)-mediated dUTP nick end labeling (TUNEL) assay. After the paraffin-embedded sections were dewaxed and dehydrated, apoptosis was detected using the TUNEL detection kit (Beyotime Institute of Biotechnology). The sections were incubated with protease $\mathrm{K}$ (diluted with $10 \mathrm{mM}$ Tris- $\mathrm{HCl}$ ) at $37^{\circ} \mathrm{C}$ for $25 \mathrm{~min}$. Following 3 PBS washes and drying, the sections were added to mounting fluid and incubated at room temperature for $20 \mathrm{~min}$. The sections were then washed again with PBS in triplicate and incubated with $50 \mu \mathrm{l}$ of biotin labeling solution at $37^{\circ} \mathrm{C}$ for $60 \mathrm{~min}$. Thereafter, the sections were washed 3 times with PBS and 2,4-diaminobutyric acid reagent was added to observe coloration under the BX-51 optical microscope (Olympus Optical Co., Ltd.). Color development was terminated by adding water, and the nuclei were stained in hematoxylin at room temperature for $1 \mathrm{~min}$, dehydrated and cleared, and were then sealed with neutral gum. Each section was observed by two pathologists under a BX-51 optical microscope (Olympus 
Optical Co., Ltd.) and the number of TUNEL-positive cells stained brown yellow in 5 random fields was counted.

Hoechst 33258 staining. The paraffin-embedded sections were dewaxed with xylene and gradient ethanol, bathed at $97^{\circ} \mathrm{C}$ in citrate buffer for $15 \mathrm{~min}$, and washed twice with PBS, 3 min each time. The sections were then stained with Hoechst 33258 dye (Invitrogen; Thermo Fisher Scientific, Inc.) at room temperature for $5 \mathrm{~min}$, and were then washed twice with PBS (3 min/time) following dye removal. A drop of anti-quenching sealing liquid was added to the sections, which were then covered with a clean cover slip while avoiding bubbles as much as possible. Finally, the sections were photographed (excitation wavelength, $350 \mathrm{~nm}$; emission wavelength, $460 \mathrm{~nm}$ ) under a BX-60 fluorescence microscope (Olympus Corporation).

Fluoro-Jade $C$ (FJC) staining. Hippocampal tissue sections were immersed in $1 \% \mathrm{NaOH}-80 \%$ ethanol mixture for $5 \mathrm{~min}$, transferred to $70 \%$ ethanol for $2 \mathrm{~min}$, and then immersed in distilled water for $2 \mathrm{~min}, 0.06 \%$ potassium permanganate solution for $10 \mathrm{~min}$, followed by washing in distilled water for $2 \mathrm{~min}$. Additionally, the sections were evenly dripped with $0.0001 \%$ FJC dye (containing $0.1 \%$ acetic acid; Chemicon International, Inc.) at room temperature for $10 \mathrm{~min}$ and were then rinsed 3 times in distilled water for $1 \mathrm{~min}$ each time. The slices were dried, cleared with xylene for $1 \mathrm{~min}$, and sealed with neutral gum. The blue filter (excitation wavelength 450-490 nm) was used to observe and collect images under the BX-60 fluorescence microscope (Olympus Corporation).

Thiobarbituric acid (TBA) method and water-soluble tetrazolium salt-8 (WST-8) method. The hippocampus tissue homogenate was centrifuged at $2,000 \mathrm{x} \mathrm{g}$ and $4^{\circ} \mathrm{C}$ for 15 min with the supernatant absorbed. The malondialdehyde (MDA) content and superoxide dismutase (SOD) activity were determined using the TBA method and WAT- 8 method, respectively, according to the manufacturer's instructions (Beyotime Institue of Biotechnology).

TEM observation. The hippocampal tissue sections at $1 \mathrm{~mm}^{3}$ were fixed in $40 \mathrm{~g} / 1$ of glutaraldehyde for $1 \mathrm{~h}$ and washed 3 times with $0.1 \mathrm{~mol} / \mathrm{l}$ of phosphate buffer ( $\mathrm{pH}$ 7.4) for $5 \mathrm{~min}$ each time. The tissue sections were fixed with $1 \%$ osmium acid for 2-3 $\mathrm{h}$ and were washed 3 times with $0.1 \mathrm{~mol} / \mathrm{l}$ of phosphate buffer ( $\mathrm{pH}$ 7.4) for $5 \mathrm{~min}$ each time. The tissue sections were dehydrated by gradient ethanol, immersed in a mixture of acetone and an equal amount of Epon812 for $3 \mathrm{~h}$, embedded in Epon812, and polymerized at $60^{\circ} \mathrm{C}$ for $48 \mathrm{~h}$. Thereafter, the sections were stained with $3 \%$ uranium acetate and lead citrate (all from Sangon Biotech) at room temperature for $15 \mathrm{~min}$, respectively, and were observed and photographed under a TEM (JEM-1200 EX; JEOL, Ltd.).

Detection of mitochondrial transmembrane potential (MTP) by JC-10 staining. The MTP in hippocampal homogenates was detected using JC-10 fluorescent probes (Beijing Solarbio Science \& Technology Co., Ltd.). The hippocampal homogenates were incubated with $500 \mu \mathrm{l}$ of $\mathrm{JC}-10$ dye solution at $37^{\circ} \mathrm{C}$ for $20 \mathrm{~min}$. Following incubation, the sections were centrifuged at $600 \mathrm{x} \mathrm{g}$ and $4^{\circ} \mathrm{C}$ for $4 \mathrm{~min}$ and the supernatants were discarded, and the sections were then washed twice with JC-10 staining buffer. The MTP was measured using a flow cytometer (Guava, Easycyte ${ }^{\mathrm{TM}}$ 8; EMD Millipore) and a Guava PCA flow cytometry system (EMD Millipore) following suspension.

MitoSOX-based flow cytometry to detect mitochondrial reactive oxygen species ( $R O S)$. The hippocampal homogenates were incubated in $0.01 \mu \mathrm{m}$ MitoSOX dye (Invitrogen; Thermo Fisher Scientific, Inc.) diluted in PBS at a ratio of 1:1 for $30 \mathrm{~min}$ at $37^{\circ} \mathrm{C}$. The supernatant was then removed, and the cells were detached using ethylene diamine tetraacetic acid-free trypsin, followed by centrifugation at $1,000 \mathrm{x} \mathrm{g}$ and $4^{\circ} \mathrm{C}$ for $5 \mathrm{~min}$. Finally, the cells were washed 3 times with $200 \mathrm{ml}$ of PBS and the ROS content was detected by flow cytometry (Guava, Easycyte ${ }^{\mathrm{TM}}$ 8; EMD Millipore) with the Guava PCA flow cytometry system (EMD Millipore).

Dual luciferase reporter gene assay. Bioinformatics software TargetScan (http://www.targetscan.org) was used to predict the targeting association and binding sites between miR-142 and PINK1. The PINK1 3'UTR promoter sequence containing the binding site of miR-142 was synthesized and inserted into the pMIR-REPORT ${ }^{\mathrm{TM}}$ Luciferase vector plasmid (Ambion, Inc.) to construct the PINK1 3'UTR wild-type (PINK1-WT) plasmid and PINK1 3'UTR mutant type (PINK1-MUT) plasmid. Plasmid extraction was carried out according to the manufacturer's instructions (Promega Corporation). PINK1-WT and PINK1-MUT were mixed with $100 \mathrm{nM}$ mimic NC and mimic miR-142 (GenePharma), respectively, and were then co-transfected into 293T cells [American Type Culture Collection (ATCC)] using Lipofectamine ${ }^{\mathrm{TM}} 2000$ (Invitrogen; Thermo Fisher Scientific, Inc.). After $48 \mathrm{~h}$, the luciferase activity was detected using a luciferase detection kit (BioVision, Inc.) and a Glomax 20/20 luminometer fluorescence detector (Promega Corporation). The ratio of firefly luciferase activity to Renilla luciferase activity was calculated as the relative activity. The experiment was repeated 3 times.

Statistical analysis. Statistical analysis was conducted using SPSS 21.0 (IBM Corp.). All the data were examined using the Kolmogorov-Smirnov test. Measurement data are expressed as the means \pm standard deviation. A t-test was used for comparisons between 2 groups, and one-way analysis of variance (ANOVA) or two-way ANOVA was used for comparisons among multiple groups. Tukey's multiple comparisons test was used as a post hoc test. P-values were obtained by a two-tailed test, and $\mathrm{P}<0.05$ was considered to indicated a statistically significant difference.

\section{Results}

Epileptic rats exhibit obvious epileptiform discharges, spikes and sharp waves. The normal rats exhibited normal behavior and no epileptic seizures. According to the Racine grading standard, epileptic seizures in epileptic rats reached grade IV-V. EEG mapping results revealed that the normal rats had no cluster spike discharges, while the epileptic rats exhibited high cluster amplitudes and high-frequency multi-spike discharges (Fig. 1). These manifestations suggested the epilepsy model was successfully established. 
A
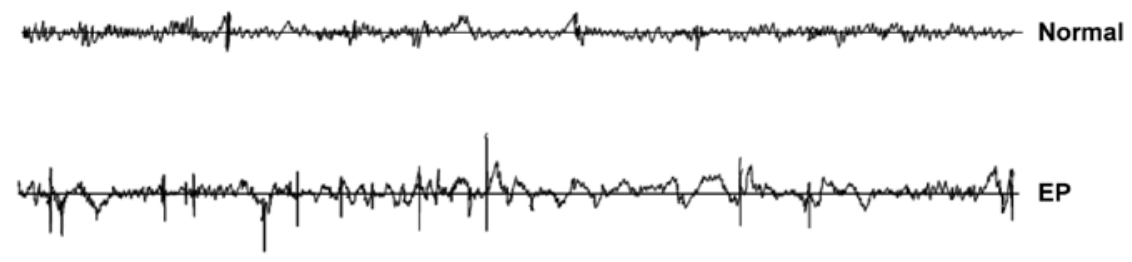

B
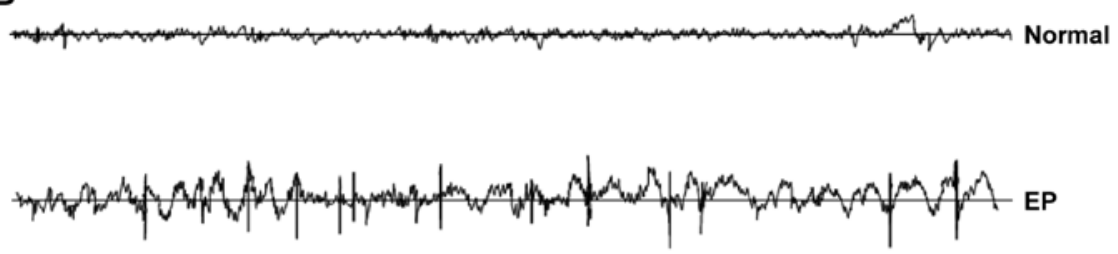

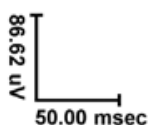

Figure 1. Successful establishment of the model of epilepsy. (A) EEG result of the cortical electrode in rats; (B) EEG result of the hippocampal electrode in rats. 'Normal' indicates results in normal rats, showing no obvious paroxysmal rhythm and epileptic discharge; 'EP' indicates results in epileptic rats $3 \mathrm{~h}$ after the epileptic seizure, showing obvious epileptic discharges, spikes and sharp waves. $\mathrm{n}=6$. EEG, electroencephalogram.

miR-142 inhibition reduces ROS generation and apoptosis in hippocampal neurons in epileptic rats. Following the successful establishment of the model of epilepsy, miR-142 expression was measured in rats subjected to different treatments. miR-142 expression was upregulated in the epileptic rats compared with that in normal rats, and was decreased in rats treated with antagomir-miR-142 compared that in rats treated with antagomir-NC (both $\mathrm{P}<0.01$; Fig. $2 \mathrm{~A}$ ). $\mathrm{H} \& \mathrm{E}$ staining (Fig. 2B) and Nissl staining (Fig. 2C) revealed that the hippocampal neurons in the normal rats were in band distribution. A number of dense vertebral cells were observed in the CA1 and CA3 regions, which were well arranged, complete in shape, clear in the edge and nucleolus, transparent in the cytoplasm, round or oval in the nucleus, and uniform in chromatin distribution, with a normal shape and abundant Nissl bodies in the cytoplasm. Additionally, the hippocampus of the epileptic rats exhibited obvious neuronal degeneration, cell structure disintegration, edema and proliferation in astrocytes. In the CA1 and CA3 regions, the structure of nerve cells was incomplete, with swelling and ruptures, a blurred outline and unclear boundaries, disordered arrangement, enlarged cell spacing, chromatin clumping in the edges, cytoplasmic edema, and enlargement of cell bodies and decreased cytoplasmic Nissl bodies. Following antagomir-miR-142 treatment, most of the pyramidal cells in the CA1 and CA3 regions of the hippocampus of rats had clear boundaries and normal morphology, with broken structures, blurred contours and unclear boundaries in a few neurons.

In the CA3 region of the hippocampus of the normal rats, only a few TUNEL-positive cells (dark brown cells; Fig. 2D) and FJC-positive cells (Fig. 2E) were observed. In the epileptic rats, the number of TUNEL-positive cells in the hippocampal CA3 region significantly increased $(\mathrm{P}<0.01$; Fig. 2D) and the bright yellow-green fluorescent FJC-positive cells with a neuron-like shape were observed with clear cell bodies and neurites. Hoechst 332528 staining (Fig. 2F) revealed that the nuclei of apoptotic cells in epileptic rats exhibited coagulation and bright blue fluorescence compared with the normal rats. Following antagomir-miR-142 treatment, the number of TUNEL-positive apoptotic cells and FJC-positive cells decreased significantly in the hippocampal CA3 region in the rats, and the numbers of cells with bright blue fluorescent decreased $(\mathrm{P}<0.01)$.

Under the TEM (Fig. 2G), the structure of hippocampal neurons in normal rats was clear and complete, the nuclear structure was normal, the nuclear pore complex was clear, chromatin was evenly distributed, and the nucleolus was located in the middle. In hippocampal neurons of epileptic rats, the cell body was shrunken, and there were small and irregular clots and chromatin margination, rough endoplasmic reticulum and mitochondrion swelling, the loss of mitochondrial cristae, organelle reduction, structural destruction, the formation of cavity and vacuoles of different sizes in the cytoplasm, and cell membrane rupture. However, these morphologies were reversed by antagomir-miR-142. Compared with the normal rats, the MDA content increased, SOD activity decreased significantly (Fig. $2 \mathrm{H}$ ), the expression of Bax in the hippocampus increased significantly, and Bcl-2 protein decreased significantly in the epileptic rats (all $\mathrm{P}<0.01$; Fig. 2I). Following the inhibition of miR-142 expression, the above-mentioned trends were all reversed.

miR-142 targets PINK1. These results indicated that miR-142 was involved in the biological process of epileptic rats in the current research. Subsequently, the present study wished to identify its downstream target gene. Using online analysis software, a specific binding region was found between the PINK1 sequence and miR-142 sequence (Fig. 3A), indicating that PINK1 is the target gene of miR-142. The dual luciferase report gene assay was used to further verify the results. Compared with the NC group, the luciferase activity of the WT-PINK1/miR-142 co-transfection group decreased 
A

Db
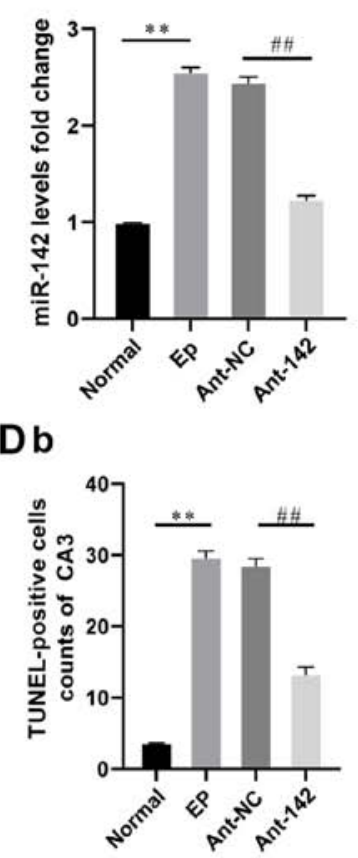

B Normal

E

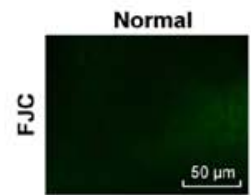

$\mathbf{F}$

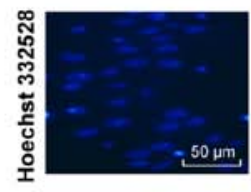

G

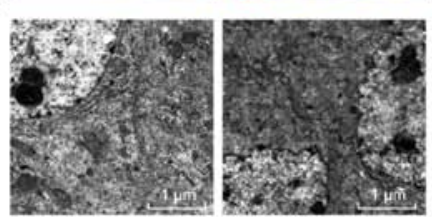

I
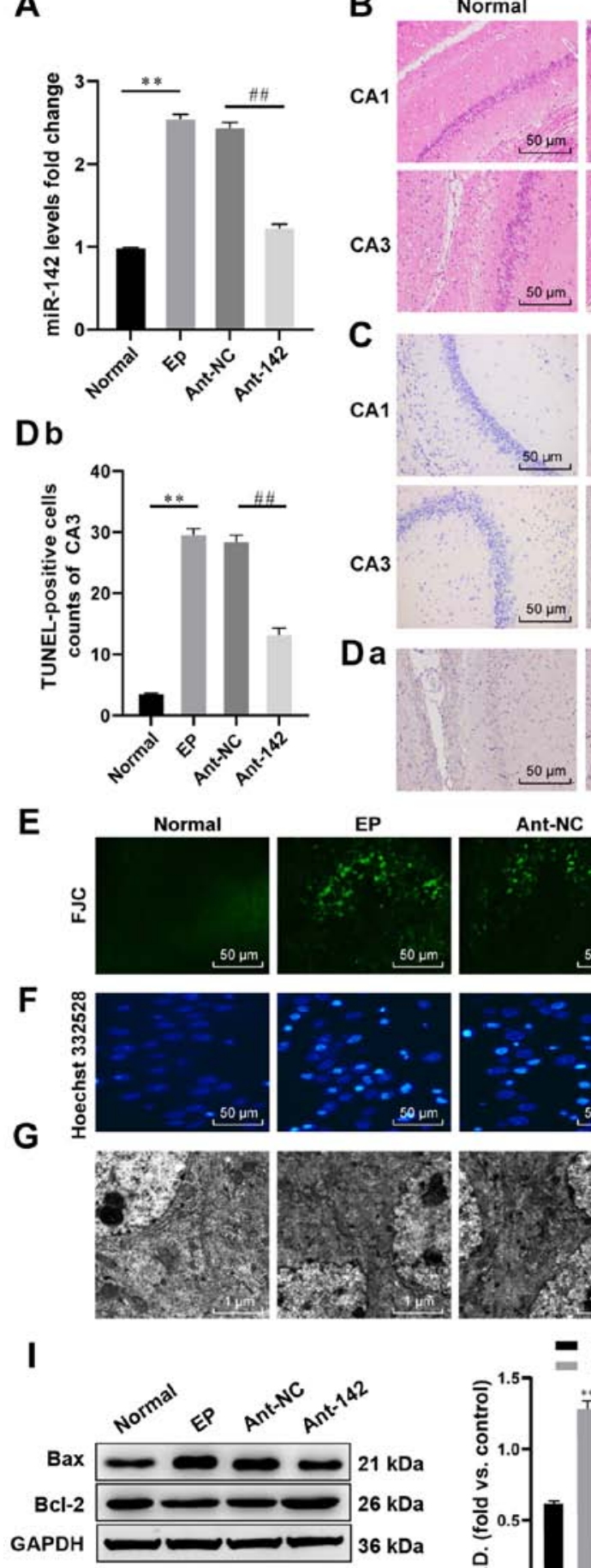

C
CA1

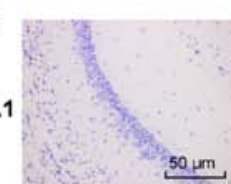

CA3

Da

EP
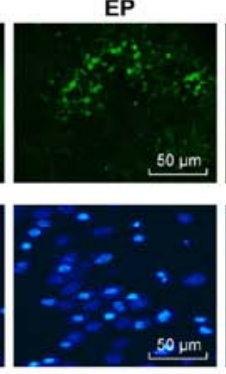
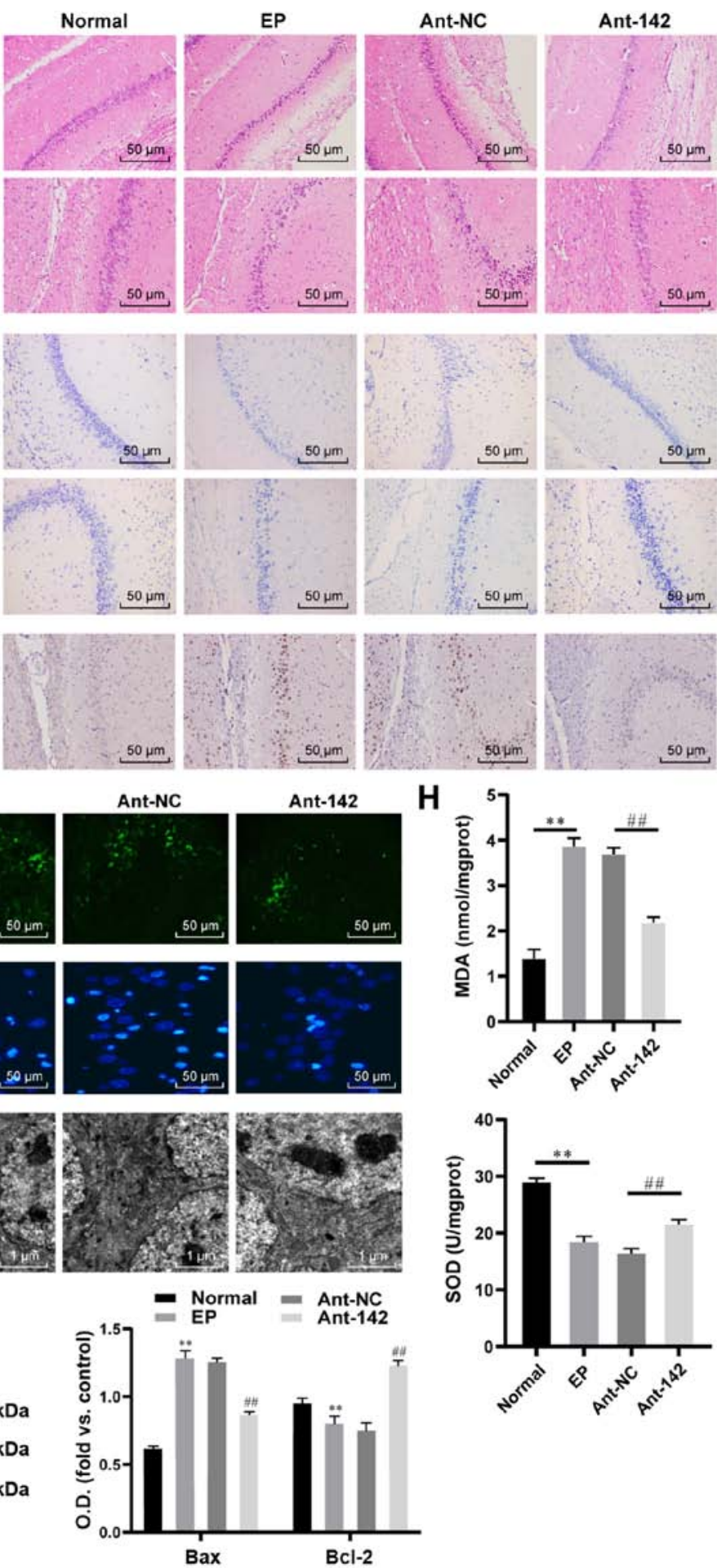

Figure 2. miR-142 inhibition reduces ROS and apoptosis in hippocampal neurons in epileptic rats. (A) Relative miR-142 expression in normal rats and epileptic rats measured by RT-qPCR, $\mathrm{n}=3$. (B and C) Representative images of the histopathological sections of the hippocampus in epileptic rats detected by H\&E staining and Nissl staining, $n=6$. (D-F) Representative images of apoptosis and degeneration in the hippocampal CA3 region of epileptic rats detected by the TUNEL assay, FJC staining and Hoechst 332528 staining, $n=6$. (G) Representative images of the neuron structure in the hippocampus of epileptic rats observed under TEM, $n=3$. (H) Relative MDA and SOD contents in normal rats and epileptic rats measured by the TBA and WST-8 methods, $n=3$. (I) Protein levels of $\mathrm{Bcl}-2$ and $\mathrm{Bax}$ in the hippocampus of normal rats and epileptic rats measured by western blot analysis, $\mathrm{n}=3$. The data were analyzed using one-way ANOVA and Tukey's multiple comparisons test as a post hoc test. ${ }^{* *} \mathrm{P}<0.01$, compared with the normal group; ${ }^{\# \#} \mathrm{P}<0.01$, compared with the antagomir-NC group. miR-142, microRNA-142; ROS, reactive oxygen species; RT-qPCR, reverse transcription-quantitative polymerase chain reaction; TUNEL, terminal deoxynucleotidyl transferase (TdT)-mediated dUTP nick end labeling; H\&E, hematoxylin and eosin; FJC, Fluoro-Jade C; MDA, malondialdehyde; SOD, superoxide dismutase; TBA, thiobarbituric acid; WST-8, water-soluble tetrazolium salt-8; Bcl-2, B-cell lymphoma-2; Bax, Bcl-2-associated X; ANOVA, analysis of variance.

$(\mathrm{P}<0.05)$, while that of MUT-PINK1 exhibited no significant difference $(\mathrm{P}>0.05)$, indicating that miR-142 could specifically bind to PINK1 (Fig. 3B). Subsequently, miR-142 mimic was constructed and the overexpression efficiency was detected. The results revealed that the overexpression of miR-142 significantly decreased the mRNA and protein levels of PINK1 (Fig. 3C). 
A

\begin{tabular}{|c|c|c|c|c|c|}
\hline $\begin{array}{l}\text { Position 748-755 of PINK1 3' UTR } \\
\text { hsa-miR-142-5p }\end{array}$ & 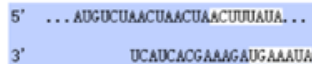 & IIIIIII & $8 \mathrm{me}$ & -0 & 9 \\
\hline
\end{tabular}

B

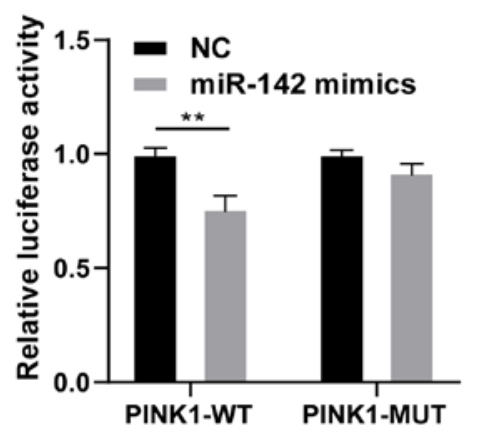

C b

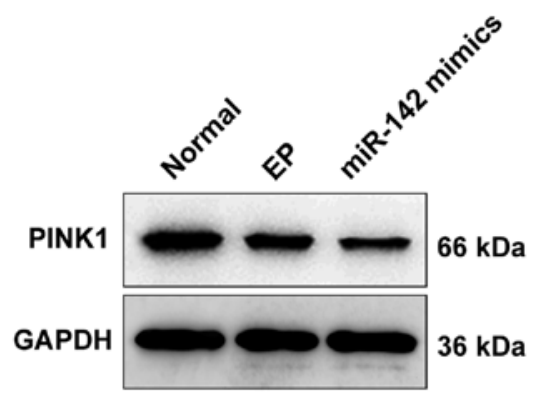

C a
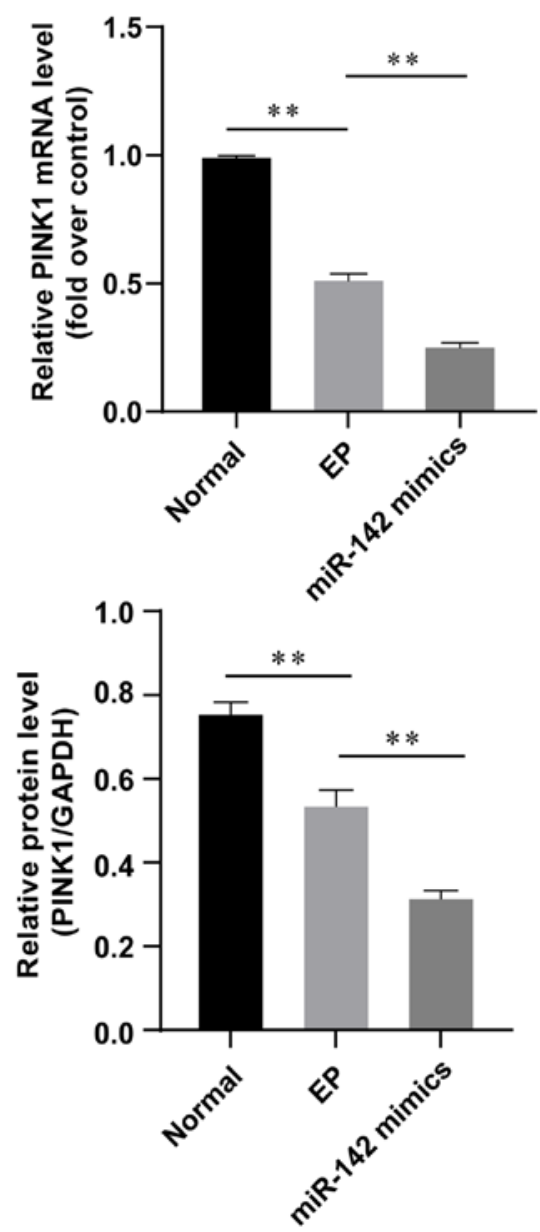

Figure 3. PINK1 is the target gene of miR-142. (A) Binding sequence of miR-142 and PINK1 was analyzed using bioinformatics software. (B) The targeting association between miR-142 and PINK1 was validated by the dual luciferase report gene assay. The data were analyzed by two-way ANOVA, and the pairwise comparisons following ANOVA were analyzed by Sidak's multiple comparisons test. ${ }^{* *} \mathrm{P}<0.01$, compared with the NC group. (C) Relative levels of PINK1 were detected by (a) RT-qPCR and (b) western blot analysis, n=3. Data were analyzed by one-way ANOVA, and pairwise comparisons following ANOVA were analyzed by Tukey's multiple comparisons test. ${ }^{* *} \mathrm{P}<0.01$, compared with the epilepsy group. miR-142, microRNA-142; PINK1, phosphatase and tensin homolog deleted on chromosome 10 (PTEN)-induced putative kinase 1; RT-qPCR, reverse transcription-quantitative polymerase chain reaction; ANOVA, analysis of variance; $\mathrm{NC}$, negative control.

Overexpression of PINK1 attenuates hippocampal damage in epileptic rats. As PINK1 is the target gene of miR-142, it may also affect the behavior of epileptic rats. Thus, the gain- and loss-of-functions of PINK1 were investigated to determine its roles in epilepsy. LV-PINK1 transfection significantly upregulated PINK1 expression in the epileptic rats, while the epileptic rats treated with anti-miR-142 and PINK1 together exhibited a downregulated PINK1 expression (both $\mathrm{P}<0.05$; Fig. 4A). Through H\&E staining (Fig. 4B) and Nissl staining (Fig. 4C), it was observed that the neurons in the epileptic rats injected with LV-PINK had a complete morphology, a distinct structure, a normal nuclear structure, a regular arrangement of vertebral cells, abundant Nissl bodies in the cytoplasm, and fewer TUNEL-positive cells (Fig. 4D) and FJC-positive cells (Fig. 4E) (all $\mathrm{P}<0.05)$. Hoechst 33258 nuclear staining (Fig. 4F) revealed that most of the cells displayed uniform light blue fluorescence and only a few apoptotic cells were observed. The inhibition of miR-142 and PINK1 together revealed that some neurons in the hippocampus were damaged more severely and were arranged disorderly and sparsely, with reduced vertebral cells and Nissl bodies, and increased TUNEL-positive cells, FJC-positive cells and bright blue cells. Briefly, the overexpression of PINK1 attenuated hippocampal damage in epileptic rats and PINK1 downregulation suppressed the protective effects of antagomir-miR-142.

miR-142 inhibition promotes mitochondrial autophagy in the hippocampal tissue of epileptic rats by targeting PINK1. The above-mentioned findings revealed that miR-142 downregulated PINK1 expression, and it has been shown that PINK1 can promote mitochondrial autophagy (20). Therefore, the present study further investigated the regulatory effects of miR-142 on PINK1-mediated mitochondrial autophagy in epileptic rats. Compared with the normal rats, the number of mitochondria in the epileptic rats decreased and many damaged mitochondria were encapsulated by the bilayer membrane. The mitochondria 


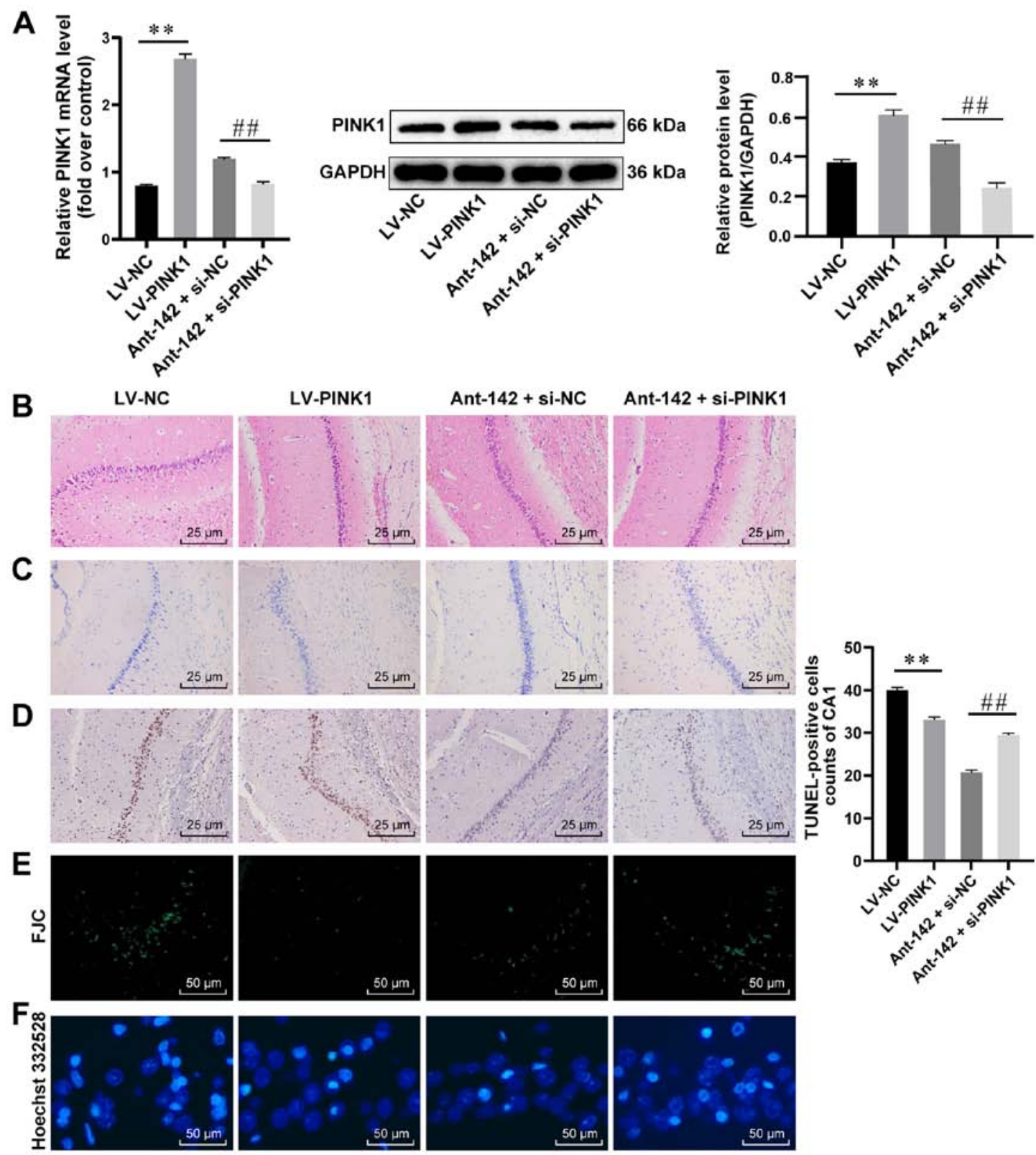

Figure 4. PINK1 overexpression is protective for hippocampal neurons in epileptic rats. (A) Relative mRNA and protein levels of PINK1 in the hippocampus of epileptic rats detected by RT-qPCR and western blot analysis, $n=3$. (B-F) Representative images of H\&E staining, Nissl staining, TUNEL assay, EJC staining and Hoechst 33258 nuclear staining, $\mathrm{n}=6$. Data were analyzed by one-way ANOVA, and the pairwise comparisons following ANOVA were analyzed by Tukey's multiple comparisons test. ${ }^{* *} \mathrm{P}<0.01$, compared with the LV-NC group; ${ }^{\# /} \mathrm{P}<0.01$, compared with the antagomir-miR-142 + siRNA-NC group. miR-142, microRNA-142; PINK1, phosphatase and tensin homolog deleted on chromosome 10 (PTEN)-induced putative kinase 1; RT-qPCR, reverse transcription quantitative polymerase chain reaction; H\&E, hematoxylin and eosin; ANOVA, analysis of variance; LV, lentivirus vector; NC, negative control.

were swollen, and the mitochondrial ridge was disordered, broken or disappeared with obvious vacuolar degeneration. Mitochondrial morphology was restored without vacuole mitochondria following the inhibition of miR-142 expression or the overexpression of PINK1 in the epileptic rats. However, mitochondrial morphology and structure were destroyed with severe vacuolar degeneration following interference with miR-142 and PINK1 (Fig. 5A).

The protein levels of C-III core 1, COX IV, TIM23 and MFN1 were upregulated in the epileptic rats; however, opposite trends were observed following the inhibition of miR-142 expression or the overexpression of PINK1. Additionally, intervention with miR-142 and PINK1 further increased the levels of the above-mentioned proteins in the epileptic rats (all $\mathrm{P}<0.01$; Fig. 5B).
In the epileptic rats, MTP decreased significantly and the number of green fluorescent cells increased, indicating that increased numbers of cells were apoptotic, while orange fluorescence exhibited normal living cells, and ROS generation increased significantly. The inhibition of miR-142 expression or the overexpression of PINK1 maintained MTP and reduced ROS generation in the epileptic rats, effects that were reversed by combined intervention with miR-142 and PINK1 (all $\mathrm{P}<0.01$; Fig. 5C and D).

\section{Discussion}

Despite several new improvements in this field over the past years, $\sim 30 \%$ of all epilepsy cases remain refractory to 
A
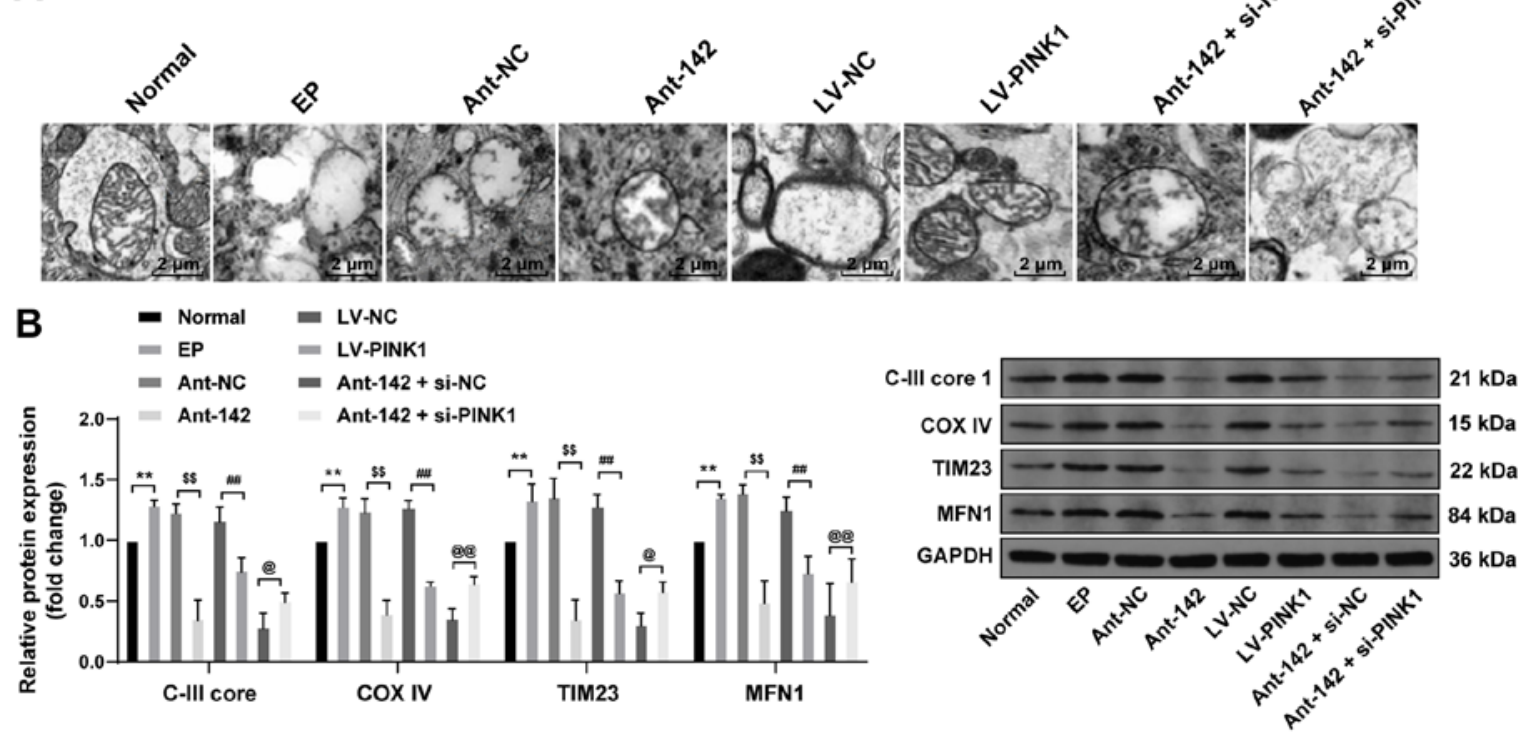

C
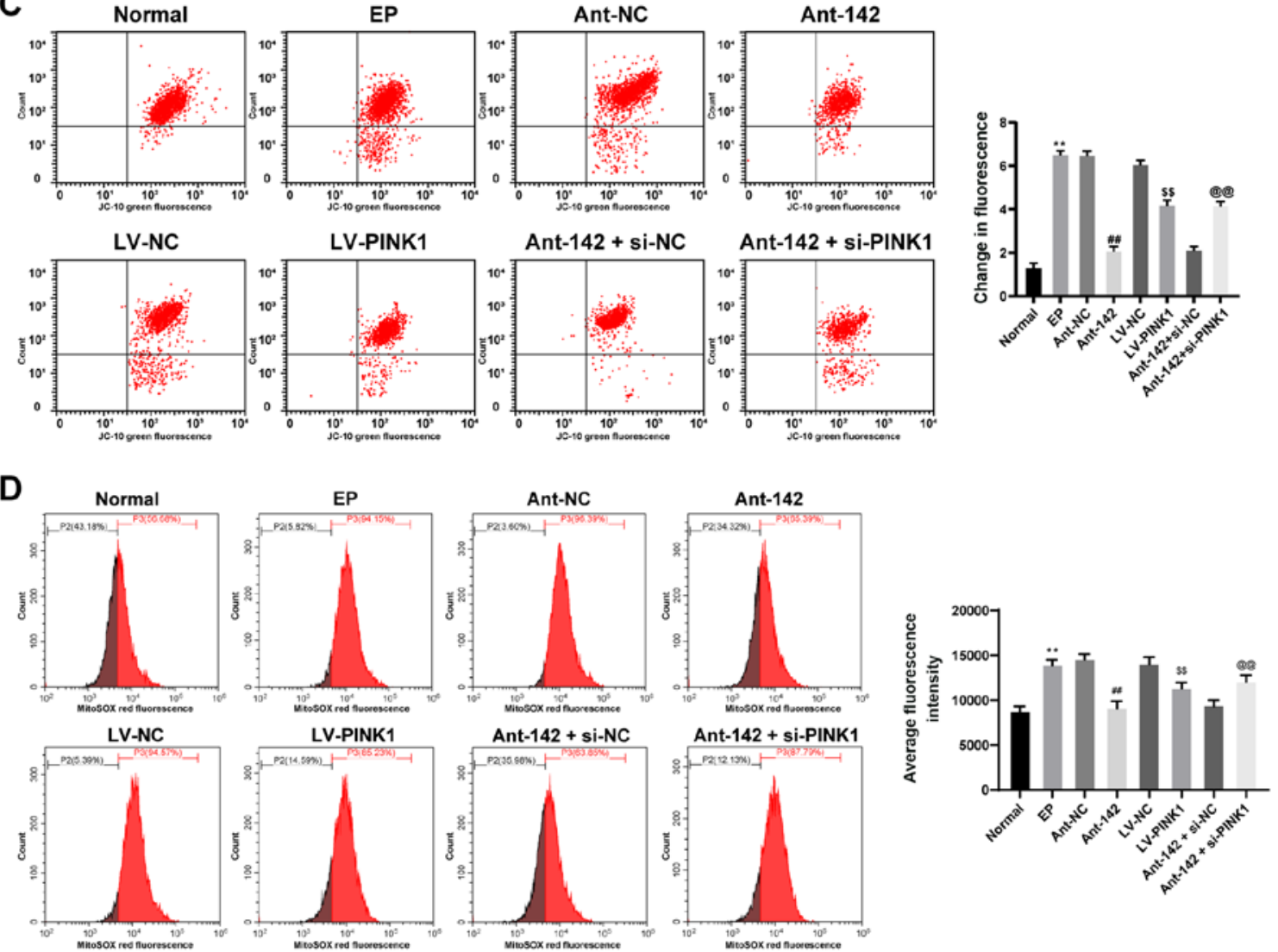

Figure 5. miR-142 inhibition promotes mitochondrial autophagy in epileptic rats by upregulating PINK1. (A) Representative images of the mitochondrial structure in the hippocampus of epileptic rats were observed by TEM, n=3. (B) Protein levels of C-III core 1, COX IV, TIM23 and MFN1 in the hippocampus of epileptic rats were measured by western blot analysis, $n=3$; (C and D) Relative MTP and ROS generation in the hippocampus of epileptic rats were measured by JC-10 staining and MitoSOX-based flow cytometry, $\mathrm{n}=3$. Data were analyzed by one-way ANOVA, and pairwise comparisons following ANOVA were analyzed by Tukey's multiple comparisons test. ${ }^{* *} \mathrm{P}<0.01$, compared with the normal group; ${ }^{\# \#} \mathrm{P}<0.01$, compared with the LV-NC group; ${ }^{\$ \$} \mathrm{P}<0.01$, compared with the antagomir-NC group; ${ }^{\circledR} \mathrm{P}<0.05,{ }^{\circledR} @ \mathrm{P}<0.01$, compared with the antagomir-miR-142 + siRNA-NC group. miR-142, microRNA-142; PINK1, phosphatase and tensin homolog deleted on chromosome 10 (PTEN)-induced putative kinase 1; TEM, transmission electron microscope; COX IV, cytochrome C oxidase IV; TIM23, translocase of the inner membrane 23; MFN1, mitochondrial fusion protein 1; MTP, mitochondrial transmembrane potential; ROS, reactive oxygen species; ANOVA, analysis of variance; $\mathrm{LV}$, lentivirus vector; NC, negative control.

medication, and seizures persist even after receiving optimum drug treatment (21). It has been revealed that a series of pathophysiological changes occur in the CA1 and CA3 in regions in the hippocampus during epilepsy (22-24). Previous evidence has supported the important roles of miRNAs in hippocampal neuronal death, brain injuries, inflammation and the immune response in the pathogenesis of epilepsy (25). Therefore, the present study focused on the pathophysiological processes 
in the CA1 and CA3 regions in the hippocampus to explore the effects of miR-142 on epileptic rats. Collectively, it was concluded that miR-142 knockdown stimulated mitochondrial autophagy and alleviated hippocampal damage in epileptic rats by targeting PINK1.

First, miR-142 expression was upregulated in epileptic rats, and rats treated with antagomir-miR-142 exhibited a normal structure of hippocampal neurons, the alleviation of hippocampal damage and mitochondrial dysfunction, a decreased MDA content, an increased SOD activity, a downregulated Bax expression and an upregulated Bal-2 expression. A recent study demonstrated that changes in miR-142 expression in the brain can lead to an increased risk of Alzheimer's disease, and elevated levels of miR-142-3p are a risk factor for Alzheimer's disease (26). MDA, a key marker of oxidative stress (OS), is expressed in the process of oxidative degradation of polyunsaturated lipids (27). ROS-induced lipid peroxidation and MDA production, in turn, contribute to neurotoxicity and cell death (28). Lengthy seizure activities lead to increased ROS production, OS) and mitochondrial dysfunction, which may cause severe brain damage (29). OS and mitochondrial dysfunction destroy the balance of the intracellular environment, leading to neuroexcitability and neuronal death in epilepsy (30). OS can damage the mitochondrial respiratory chain and lead to the excessive production of ROS, the accumulation of which can inhibit the activity of the mitochondrial respiratory enzyme complex, leading to cell death in the epileptic area (16). Additionally, a reduction in OS protects the hippocampus from abnormal mossy fiber sprouting, thus lessening recurrent seizures and repressing hippocampal neuronal loss in a rat model of temporal lobe epilepsy (31). Furthermore, an increased MDA content is assocaited with the early age of epilepsy onset (32). SOD1 levels are downregulated in the cerebrospinal fluid of patients with epilepsy, particularly those with refractory epilepsy; thus, the decreased SOD1 level may predict antiepileptic drug resistance in epileptic patients (33). Consistent with the results of the present study, a previous study displayed significantly enhanced OS in the hippocampus injected with lithium chloride-pilocarpine, as evidenced by a a markedly increased MDA content and a decreased SOD activity (16). Partially coinciding with the current findings, the elevation of SOD activity and the downregulation of the MDA content has been reported to inhibit OS in Parkinson's disease, thereby protecting against dopaminergic neuronal cell apoptosis (34). Notably, a recent study revealed that, partly in line with the current observation, namely, the inhibition of miR-142-5p attenuated oxygen-glucose deprivation and reoxygenation $(\mathrm{OGD} / \mathrm{R})$-induced cell injury in hippocampal neurons and OS, indicated by decreased MDA levels and increased SOD levels, and promoted cell viability and inhibited apoptosis (35).

Furthermore, PINK1 is the target gene of miR-142, and the overexpression of PINK1 attenuated hippocampal damage in epileptic rats, and PINK1 downregulation attenuated the protective effects of antagomir-miR-142. In humans, PINK1 is highly expressed in the hippocampus, and it antagonizes mitochondrial dysfunction and protects neurons against OS and neurotoxins (36). Additionally, mice with a deficiency in PINK1 exhibit increased oxidative damage and mitochondrial dysfunction in the striatum (37). In primary cultured rat hippocampal neurons, the overexpression of PINK1 has been shown to lead to increased mitochondrial, and a diminished mitochondrial size and proportion in neuronal processes (38). Moreover, the present study found that the protein levels of C-III core 1, COX IV, TIM23 and MFN1 were upregulated, the MTP was decreased and ROS production increased significantly in epileptic rats; however, opposite trends were observed following the inhibition of miR-142 expression or the overexpression of PINK1, indicating enhanced mitochondrial autophagy. C-III core 1, COX IV, TIM23 and MFN1 are well-known indicators of mitochondrial autophagy, among which MFN1 promotes mitochondrial elongation and activity (39). PINK1 is specifically activated by MTP depolarization, and the overexpression of PINK1 enhances mitochondrial fission (40). PINK1 deficiency results in mitochondrial fragmentation, ROS production, decreased MTP and mitochondrial cristae density, and the inhibition of mitochondrial autophagy (41). Importantly, the inhibition of miR-142-5p can promote PTEN expression and increase autophagy levels (42).

Taken together, the present study demonstrates that miR-142 expression is upregulated in epileptic rats, and that miR-142 inhibition can stimulate mitochondrial autophagy and alleviate hippocampal damage and ROS in epileptic rats by targeting PINK1. Additionally, the pathological and physiological changes in the dentate gyrus area are an important characterization in epileptic rats; thus, the authors aim to conduct a further in-depth investigations on the changes in the dentate gyrus area in epileptic rats in the future. The findings of the present study may provide a novel understanding of the mechanisms of epilepsy, enabling the identification of more effective therapeutic approaches. Further research is warranted to further validate the current results and apply the results to clinical settings.

\section{Acknowledgements}

Not applicable.

\section{Funding}

No funding was received.

\section{Availability of data and materials}

The datasets used and/or analyzed during the current study are available from the corresponding author on reasonable request.

\section{Authors' contributions}

All authors are the guarantors of the integrity of the entire study. DX, JL and ZZ contributed to the study concept and design. YL and YZ contributed to the study design and experiments. $\mathrm{CLu}, \mathrm{BQ}, \mathrm{CLi}$ and $\mathrm{LQ}$ were responsible for the acquisition and analysis of data. DX, JL, ZZ, BQ and CLi contributed to the manuscript preparation and manuscript editing. All the authors read and approved the final manuscript.

\section{Ethics approval and consent to participate}

The present study was approved and supervised by the Ethics Committee of Guangzhou Women and Children's Medical 
Center. The protocol was also approved by the Institutional Animal Care and Use Committee of Guangzhou Women and Children's Medical Center. Significant efforts were made to minimize the number of animals used and their pain.

\section{Patient consent for publication}

Not applicable.

\section{Competing interests}

The authors declare that they have no competing interests.

\section{References}

1. Vezzani A, French J, Bartfai T and Baram TZ: The role of inflammation in epilepsy. Nat Rev Neurol 7: 31-40, 2011.

2. Vezzani A, Fujinami RS, White HS, Preux PM, Blümcke I, Sander JW and Löscher W: Infections, inflammation and epilepsy. Acta Neuropathol 131: 211-234, 2016.

3. Moon J, Lee ST, Choi J, Jung KH, Yang H, Khalid A, Kim JM, Park KI, Shin JW, Ban JJ, et al: Unique behavioral characteristics and microRNA signatures in a drug resistant epilepsy model. PLoS One 9: e85617, 2014

4. Newton CR and Garcia HH: Epilepsy in poor regions of the world. Lancet 380: 1193-1201, 2012.

5. Gu L, Liang B, Chen Q, Long J, Xie J, Wu G, Yan Y, Tan J, Dou W, Chen W, et al: Prevalence of epilepsy in the People's Republic of China: A systematic review. Epilepsy Res 105: 195-205, 2013.

6. Guerrini R, Marini C and Mantegazza M: Genetic epilepsy syndromes without structural brain abnormalities: Clinica features and experimental models. Neurotherapeutics 11: 269-285, 2014.

7. van Campen JS, Jansen FE, de Graan PN, Braun KP and Joels M: Early life stress in epilepsy: A seizure precipitant and risk factor for epileptogenesis. Epilepsy Behav 38: 160-171, 2014.

8. Wu M, Liu X, Chi X, Zhang L, Xiong W, Chiang SMV, Zhou D and Li J: Mitophagy in refractory temporal lobe epilepsy patients with hippocampal sclerosis. Cell Mol Neurobiol 38: 479-486, 2018.

9. Cao DD, Li L and Chan WY: MicroRNAs: Key regulators in the central nervous system and their implication in neurological diseases. Int J Mol Sci 17: 842, 2016.

10. Gorter JA, Iyer A, White I, Colzi A, van Vliet EA, Sisodiya S and Aronica E: Hippocampal subregion-specific microRNA expression during epileptogenesis in experimental temporal lobe epilepsy. Neurobiol Dis 62: 508-520, 2014.

11. Shrestha A,MukhametshinaRT,TaghizadehS,Vásquez-PachecoE, Cabrera-Fuentes H, Rizvanov A, Mari B, Carraro G and Bellusci S: MicroRNA-142 is a multifaceted regulator in organogenesis, homeostasis, and disease. Dev Dyn 246: 285-290, 2017.

12. Junker A, Krumbholz M,Eisele S, Mohan H, Augstein F, Bittner R, Lassmann H, Wekerle H, Hohlfeld R and Meinl E: MicroRNA profiling of multiple sclerosis lesions identifies modulators of the regulatory protein CD47. Brain 132: 3342-3352, 2009.

13. Lin W and Kang UJ: Structural determinants of PINK1 topology and dual subcellular distribution. BMC Cell Biol 11: 90, 2010.

14. Dagda RK, Pien I, Wang R, Zhu J, Wang KZQ, Callio J, Banerjee TD, Dagda RY and Chu CT: Beyond the mitochondrion: Cytosolic PINK1 remodels dendrites through protein kinase A J Neurochem 128: 864-877, 2014.

15. Kwak YD, Wang B, Pan W, Xu H, Jiang X and Liao FF: Functional interaction of phosphatase and tensin homologue (PTEN) with the E3 ligase NEDD4-1 during neuronal response to zinc. J Biol Chem 285: 9847-9857, 2010.

16. Sun J, Gao X, Meng D, Xu Y, Wang X, Gu X, Guo M, Shao X, Yan H, Jiang C and Zheng Y: Antagomirs targeting MiroRNA-134 attenuates epilepsy in rats through regulation of oxidative stress, mitochondrial functions and autophagy. Front Pharmacol 8: 524, 2017.

17. Racine RJ: Modification of seizure activity by electrical stimulation: Cortical areas. Electroencephalogr Clin Neurophysiol 38: $1-12,1975$
18. Liao Y, Huang Y, Liu X, Luo C, Zou D, Wei X, Huang Q and Wu Y: MicroRNA-328a regulates water maze performance in PTZ-kindled rats. Brain Res Bull 125: 205-210, 2016.

19. Livak KJ and Schmittgen TD: Analysis of relative gene expression data using real-time quantitative PCR and the 2(-Delta Delta C(T)) method. Methods 25: 402-408, 2001.

20. Ma P, Yun J, Deng H and Guo M: Atg1 mediated autophagy suppresses tissue degeneration in pink1/parkin mutants by promoting mitochondrial fission in drosophila. Mol Biol Cell 29: 3082-3092, 2018.

21. Jacobs J, Staba R, Asano E, Otsubo H, Wu JY, Zijlmans M, Mohamed I, Kahane P, Dubeau F, Navarro V and Gotman J: High-frequency oscillations (HFOs) in clinical epilepsy. Prog Neurobiol 98: 302-315, 2012

22. Feng L, Shu Y, Wu Q, Liu T, Long H, Yang H, Li Y and Xiao B: EphA4 may contribute to microvessel remodeling in the hippocampal CA1 and CA3 areas in a mouse model of temporal lobe epilepsy. Mol Med Rep 15: 37-46, 2017.

23. Liu Z, Yang C, Meng X, Li Z, Lv C and Cao P: Neuroprotection of edaravone on the hippocampus of kainate-induced epilepsy rats through Nrf2/HO-1 pathway. Neurochem Int 112: 159-165, 2018.

24. Saffarzadeh F, Eslamizade MJ, Mousavi SM, Abraki SB, Hadjighassem MR and Gorji A: TRPV1 receptors augment basal synaptic transmission in CA1 and CA3 pyramidal neurons in epilepsy. Neuroscience 314: 170-178, 2016.

25. Henshall DC: MicroRNA and epilepsy: Profiling, functions and potential clinical applications. Curr Opin Neurol 27: 199-205, 2014.

26. GhanbariM,MunshiST,MaB,LendemeijerB,BansalS,AdamsHH, Wang W, Goth K, Slump DE, van den Hout MCGN, et al: A functional variant in the miR-142 promoter modulating its expression and conferring risk of Alzheimer disease. Hum Mutat 40: 2131-2145, 2019.

27. Qiang M, Xu Y, Lu Y, He Y, Han C, Liu Y and He R: Autofluorescence of MDA-modified proteins as an in vitro and in vivo probe in oxidative stress analysis. Protein Cell 5: 484-487, 2014.

28. Roomruangwong C, Barbosa DS, de Farias CC, Matsumoto AK, Baltus TH, Morelli NR, Kanchanatawan B, Duleu S, Geffard M and Maes M: Natural regulatory IgM-mediated autoimmune responses directed against malondialdehyde regulate oxidative and nitrosative pathways and coupled with IgM responses to nitroso adducts attenuate depressive and physiosomatic symptoms at the end of term pregnancy. Psychiatry Clin Neurosci 72: 116-130, 2018.

29. Dillioglugil MO, Kir HM, Demir C, Ilbay G, Sahin D, Dillioglugil O, Bambal G, Mekik H and Ates N: Effect of pentylenetetrazole and sound stimulation induced single and repeated convulsive seizures on the MDA, GSH and NO levels, and SOD activities in rat liver and kidney tissues. Brain Res Bull 83: 356-359, 2010.

30. Chang SJ and Yu BC: Mitochondrial matters of the brain: Mitochondrial dysfunction and oxidative status in epilepsy. J Bioenerg Biomembr 42: 457-459, 2010.

31. Baluchnejadmojarad T and Roghani M: Coenzyme q10 ameliorates neurodegeneration, mossy fiber sprouting, and oxidative stress in intrahippocampal kainate model of temporal lobe epilepsy in rat. J Mol Neurosci 49: 194-201, 2013.

32. Lorigados Pedre L, Gallardo JM, Morales Chacon LM, García AV, Flores-Mendoza M, Neri-Gómez T, Díaz BE, Cruz-Xenes RM, Fuentes NP and Orozco-Suárez S: Oxidative stress in patients with drug resistant partial complex seizure. Behav Sci (Basel) 8: 59, 2018.

33. Chen D, Lu Y, Yu W, Luo J, Xiao Z, Xiao F and Wang X: Clinical value of decreased superoxide dismutase 1 in patients with epilepsy. Seizure 21: 508-511, 2012.

34. Li J, Wang LN, Xiao HL, Li X and Yang JJ: Effect of electroacupuncture intervention on levels of SOD, GSH, GSH-Px, MDA, and apoptosis of dopaminergic neurons in substantia Nigra in rats with Parkinson's disease. Zhen Ci Yan Jiu 39: 185-191, 2014 (In Chinese).

35. Wang N, Zhang L, Lu Y, Zhang M, Zhang Z, Wang $\mathrm{K}$ and Lv J: Down-regulation of microRNA-142-5p attenuates oxygen-glucose deprivation and reoxygenation-induced neuron injury through up-regulating Nrf2/ARE signaling pathway. Biomed Pharmacother 89: 1187-1195, 2017.

36. Priyadarshini M, Orosco LA and Panula PJ: Oxidative stress and regulation of Pink1 in zebrafish (Danio rerio). PLoS One 8: e81851, 2013. 
37. Narendra DP, Jin SM, Tanaka A, Suen DF, Gautier CA, Shen J, Cookson MR and Youle RJ: PINK1 is selectively stabilized on impaired mitochondria to activate Parkin. PLoS Biol 8: e1000298, 2010.

38. Yu W, Sun Y, Guo S and Lu B: The PINK1/Parkin pathway regulates mitochondrial dynamics and function in mammalian hippocampal and dopaminergic neurons. Hum Mol Genet 20: 3227-3240, 2011.

39. White JP, Baltgalvis KA, Puppa MJ, Sato S, Baynes JW and Carson JA: Muscle oxidative capacity during IL-6-dependent cancer cachexia. Am J Physiol Regul Integr Comp Physiol 300: R201-R211, 2011.

40. Kondapalli C, Kazlauskaite A, Zhang N, Woodroof HI, Campbell DG, Gourlay R, Burchell L, Walden H, Macartney TJ, Deak M, et al: PINK1 is activated by mitochondrial membrane potential depolarization and stimulates Parkin E3 ligase activity by phosphorylating Serine 65. Open Biol 2: 120080, 2012.
41. Cherra SJ III, Dagda RK, Tandon A and Chu CT: Mitochondrial autophagy as a compensatory response to PINK1 deficiency. Autophagy 5: 1213-1214, 2009.

42. Chen J, Cui Y, Zhang N, Yao X, Wang Z and Yang L: Oleanolic acid attenuated diabetic mesangial cell injury by activation of autophagy via miRNA-142-5p/PTEN signaling. Cytotechnology 71: 925-933, 2019.

This work is licensed under a Creative Commons Attribution-NonCommercial-NoDerivatives 4.0 International (CC BY-NC-ND 4.0) License. 